\title{
chaJOPEN
}

\section{Estimate of the contemporary live-birth prevalence of recurrent 22q11.2 deletions: a cross-sectional analysis from population-based newborn screening}

\author{
Christina Blagojevic BMSc, Tracy Heung MA, Mylene Theriault MSc, Aoy Tomita-Mitchell PhD, \\ Pranesh Chakraborty MD, Kristin Kernohan PhD, Dennis E. Bulman PhD, Anne S. Bassett MD
}

Abstract

Background: Although pathogenic 22q11.2 deletions are an important cause of developmental delays and lifelong disease burden, their variable and complex clinical expression contributes to under-recognition, delayed molecular diagnosis and uncertainty about prevalence. We sought to estimate the contemporary live-birth prevalence of typical $22 q 11.2$ deletions using a population-based newborn screening sample and to examine data available for associated clinical features.

Methods: Using DNA available from an unbiased sample of about $12 \%$ of all dried blood spots collected for newborn screening in Ontario between January 2017 and September 2018, we prospectively screened for 22q11.2 deletions using multiplex quantitative polymerase chain reaction assays and conducted independent confirmatory studies. We used cross-sectional analyses to compare available clinical and T-cell receptor excision circle (TREC, used in newborn screening for severe combined immunodeficiency) data between samples with and without 22q11.2 deletions.

Results: The estimated minimum prevalence of 22 q11.2 deletions was 1 in 2148 (4.7 per 10 000) live births (95\% confidence interval [Cl] 2.5 to 7.8 per 10000 ), based on a total of 30074 samples screened, with 14 having confirmed $22 q 11.2$ deletions. Of term singletons, samples with 22q11.2 deletions had significantly younger median maternal age (25.5 v. $32.0 \mathrm{yr}$, difference $-6.5 \mathrm{yr}$, $95 \% \mathrm{Cl}-7$ to $-2 \mathrm{yr}$ ), a greater proportion with small birth weight for gestational age (odds ratio $7.00,95 \% \mathrm{Cl} 2.36$ to 23.18 ) and lower median TREC levels (108.9 v. 602.5 copies $/ 3 \mu \mathrm{L}, p<0.001)$.

Interpretation: These results indicate that the 22 q11.2 deletion syndrome is one of the most common of rare genetic conditions and may be associated with relatively younger maternal ages and with prenatal growth abnormalities. The findings support the public health importance of early - prenatal and neonatal - diagnosis that would enable prompt screening for and management of wellknown actionable features associated with 22q11.2 deletions.

T he $22 \mathrm{q} 11.2$ deletion syndrome $(22 \mathrm{q} 11.2 \mathrm{DS}$; Online Mendelian Inheritance in Man [OMIM] 188400/192430), previously called DiGeorge or velocardiofacial syndrome, is an important genetic condition associated with recurrent 22q11.2 microdeletions and highly penetrant expression. ${ }^{1}$ Features include developmental delay, intellectual disability, congenital cardiac or palatal anomalies (or both), pediatric immunodeficiency, and treatable endocrinologic and neuropsychiatric conditions. Variable presentation, often without major anatomic anomalies, contributes to clinical under-recognition and diagnostic delay, often with many years before molecular diagnosis. ${ }^{1,2}$

We are unaware of any contemporary population-based live-birth prevalence estimates for 22q11.2 deletions based on newborn screening data. Prevalence estimates vary widely, most commonly reported as 1.7 to 3.3 per 10000 live births. ${ }^{1}$ Dating back to $1996,{ }^{3}$ previous estimates have used multiple strategies, including ascertainment from birth defects registries, ${ }^{3,4}$ infants with congenital cardiac disease $e^{4,5}$ or clinically indicated genetic testing results. ${ }^{6,7}$ One study used 25704 newborn screening samples selected from individuals born between 1981 and 2005 to retrospectively identify 22 q11.2 deletions, but that study excluded neonatal and early infant deaths. ${ }^{8}$ Newborn screening programs using T-cell receptor excision circles (TRECs) for identification of severe combined immunodeficiency can detect some individuals with 22q11.2DS (those with neonatal immunodeficiency ${ }^{9-12}$ ); however, phenotypically based methods are unlikely to be

Competing interests: None declared.

This article has been peer reviewed.

Correspondence to: Anne Bassett, anne.bassett@utoronto.ca CMAJ Open 2021. DOI:10.9778/cmajo.20200294 
sufficient for population-wide detection of pathogenic deletions with such variable expression. ${ }^{10,11}$

Given the morbidity and mortality associated with 22q11.2DS, which extend throughout the lifespan, ${ }^{1,13-16}$ it has been proposed that $22 \mathrm{q} 11.2$ deletions be added to newborn screening panels, a plan endorsed by families of affected individuals. ${ }^{14}$ In addition to newborn screening considerations, estimates from prenatal studies and technologic advances in prenatal screening for 22q11.2 deletions (e.g., noninvasive prenatal testing) have increased the urgency of determining the current live-birth prevalence. ${ }^{15,16} \mathrm{We}$ sought to estimate the minimum live-birth prevalence of typical 22q11.2 deletions using contemporary population-based newborn screening data. We also examined available clinical data, including TREC results.

\section{Methods}

\section{Study design and setting}

We employed a cross-sectional study design to estimate the prevalence of 22q11.2 deletions by systematically screening for the most common pathogenic 22q11.2 deletions (Figure 1) ${ }^{1}$ within a subset of prospectively collected Ontario newborn screening samples. We also aimed to compare newborn screening results for TREC and clinical variables between those with and without 22q11.2 deletions.

We used anonymized dried blood spot samples collected by Newborn Screening Ontario between January 2017 and September 2018. ${ }^{1}$ The total number of samples studied was determined by power analysis for this rare disease and by funding availability (Appendix 1, Supplemental methods, available at www.cmajopen.ca/content/9/3/E802/suppl/ DC1).

\section{Data sources}

We analyzed neonatal dried blood spot-derived samples collected as part of Newborn Screening Ontario's newborn screening program, as per the organization's policy on storage and secondary use of samples. Residual DNA from the TREC quantitative real-time polymerase chain reaction (qPCR) assay, a part of Ontario's newborn screening program for severe combined immunodeficiency, ${ }^{18}$ was saved for use in this study and was available for all collected samples (i.e., there were no exclusions). Clinical data for the neonates screened were entered at the point of care.

\section{Laboratory investigations and clinical variables}

The primary qPCR screening assay for $22 \mathrm{q} 11.2$ deletions comprised primers and probes for three 22q11.2 deletion region genes (Figure 1): UFD1L and COMT (located in the low copy repeat LCR22A-LCR22B region) and CRKL (located in the LCR22C-LCR22D region), with RPPH1 used as a reference gene for appropriate DNA extraction and relative quantification (Appendix 1, Supplemental methods, Table S1 and Table S2). For each probe, the relative quantification value was calculated and a cut-off value defined using the area under the receiver operating characteristic curve
(Appendix 1, Supplemental methods, Table S3). A screenpositive sample was defined as having a putative deletion of all three 22q11.2 region probes (i.e., a suspected LCR22ALCR22D deletion) or deletion of both the UFD1L and COMT probes but not the CRKL probe (i.e., a suspected LCR22A-LCR22B or LCR22A-LCR22C deletion; see Figure 1). ${ }^{1}$ Screen-positive samples with sufficient DNA for an additional dried blood spot punch were then subjected to a secondary qPCR screening assay using the same reference probe but a different 22q11.2 probe (TBX1; Appendix 1, Supplemental methods, Table S3).

Initially, the primary screen-positive samples that also had a TBX1 relative quantification value below (or near) an established cut-off (Appendix 1, Supplemental methods, Table S3) $)^{19}$ were prioritized for standard multiplex ligationdependent probe amplification (MLPA) assays (MRC Holland, Amsterdam, Netherlands); 6 screen-negative samples served as controls for MLPA normalization (Appendix 1, Supplemental methods). Subsequently, the remainder of the screen-positive samples were subjected to MLPA confirmatory testing (Appendix 1, Supplemental methods). Samples were deemed to have a confirmed 22q11.2 deletion if they screened positive on the initial 3-probe qPCR assay and if MLPA determined the presence of a common or proximal nested 22q11.2 deletion (Figure 1). Methodologic details, including DNA extraction and creation of dried blood spot quality control material for all qPCR and MLPA assays, are provided in Appendix 1, Supplemental methods.

Available clinical variables were confined to maternal age, newborn sex, birth weight, gestational age, neonatal transfusion status and neonatal feeding type. Sample size varied for each variable because of missing data. We inspected the data for outliers and excluded any data points that appeared to be possible data entry errors.

\section{Statistical analysis}

We calculated a live-birth minimum prevalence estimate of the $22 \mathrm{q} 11.2$ deletion by dividing the number of newborn screening samples with MLPA-confirmed 22q11.2 deletion by the total number of newborn samples assessed. We calculated $95 \%$ confidence intervals (CIs) for prevalence using the binomial distribution. For the subgroup of singleton newborns born at term (defined as $\geq 37 \mathrm{wk}$ and $<42 \mathrm{wk}$ gestational age $\mathrm{e}^{20}$ ), we compared TREC values and other available clinical variables between those with a confirmed $22 \mathrm{q} 11.2$ deletion and the remaining population-based sample using the $\chi^{2}$ or Fisher exact test for categorical variables and the Mann-Whitney $U$ test for non-normally distributed continuous data. For the clinical variables, we analyzed only data for the singleton term newborns because multiplegestation and preterm births are likely to affect the variables studied, such as TREC levels. ${ }^{12}$ We also calculated $95 \%$ CIs for differences between medians, and we calculated odds ratios with $95 \%$ CIs for proportions. ${ }^{21}$

We performed statistical analyses using SAS software, version 9.4 (SAS Institute) and R statistical software (version 4.0.2). We defined statistical significance as $p<0.05,2$-tailed. 


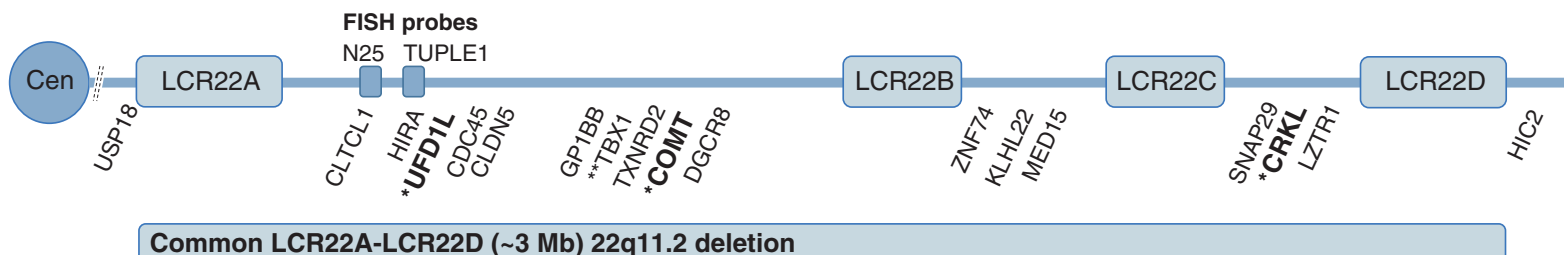

Nested LCR22A-LCR22C ( 2 Mb) 22q11.2 deletion

Nested LCR22A-LCR22B ( 1.5 Mb) 22q11.2 deletion

Figure 1: Illustration of the common 22q11.2 deletion (about 3 megabases [Mb]) and the rarer proximal nested 22q11.2 deletions (about $2 \mathrm{Mb}$ and about $1.5 \mathrm{Mb}$ ), as well as the approximate positions of genes for probes used to detect these deletions: 3 primary screening quantitative real-time polymerase chain reaction (qPCR) probes (UFD1L, COMT, CRKL, bold font, single asterisk), 15 genes for confirmatory multiplex ligation-dependent probe amplification studies (16 probes, including 2 at TBX1, and flanking probes at USP18 and HIC2), and 1 secondary screening qPCR probe (TBX1, 2 asterisks). Also shown are the relative positions of the low copy repeat (LCR) sequences (segmental duplications) that predispose this complex genomic region to de novo 22q11.2 deletion events at gametogenesis, and probes (N25 and TUPLE1) commonly used for targeted fluorescence in situ hybridization (FISH) studies that cannot determine the length of deletions. Clinical genome-wide microarray, the current standard for detecting pathogenic copy number variation, ${ }^{1,17}$ provides information on deletion length and extent. Note: Cen = centromere.

\section{Ethics approval}

This study was approved by the Children's Hospital of Eastern Ontario Research Ethics Board and the Centre for Addiction and Mental Health Research Ethics Board. In accordance with guidelines from these bodies regarding storage and secondary use of dried blood spot samples that are designed to ensure that the samples remain unidentifiable, minimal clinical data for the newborns were available.

\section{Results}

A total of 30074 anonymized dried blood spot samples collected by Newborn Screening Ontario were available for study, corresponding to $12 \%$ of all infants born in Ontario, Canada, during the 21-month period of data collection. Because of intermittent sampling, nearly all of these samples (30 017/30 074 [> 99\%]) were from infants born in 11 of the 21 months. For the overall sample, available clinical data showed that 14662 (49\%) were known females, 29087 (97\%) were known singletons, and 987 (3\%) were known multiple births. The infants were born at mean gestational age 39 (standard deviation 2) weeks, with a total of 2595 (9\%) being preterm. For the subsample of 29087 singletons, 2002 were preterm births, 64 were post-term births, and 573 had no gestational age data available; thus, there were a total of 26448 singleton term births.

\section{Estimated live-birth prevalence of the 22q11.2 deletion}

Screening and confirmatory assay results provided a minimum estimate of live-birth prevalence of the pathogenic 22q11.2 deletion of 1 in 2148 ( 4.7 per $10000,95 \%$ CI 2.5 to 7.8 per
10 000), based on 30074 Ontario newborn screening samples. There were 14 samples that had screen-positive results on the primary qPCR assay and that had MLPA confirmation (Appendix 1, Supplemental results): 11 (79\%) with the common LCR22A-LCR22D 22q11.2 deletion and 3 (21\%) with proximal nested deletions (2 LCR22A-LCR22B and 1 LCR22A-LCR22C) (Figure 1).

\section{Clinical variables}

All 14 newborn screening samples with a 22q11.2 deletion were singleton births. There were 13 term births and 1 preterm birth. Of the total 26448 singleton term births, we compared the 13 with a $22 \mathrm{q} 11.2$ deletion to the remaining population sample who had available data for sex, birth weight and gestational age $(n=26305)$. Table 1 summarizes the results (see footnotes for details of sample sizes for each variable).

Median maternal age (with interquartile range [IQR]) was significantly younger for those with a $22 \mathrm{q} 11.2$ deletion (25.5 [IQR 24.0-29.5] yr v. 32.0 [IQR 28.0-35.0] yr; difference $-6.5 \mathrm{yr}, 95 \% \mathrm{CI}-7$ to $-2 \mathrm{yr}$; Table 1$)$. Those with a $22 \mathrm{q} 11.2$ deletion also had a higher prevalence of low $(<10$ th percentile) birth weight for gestational age (odds ratio [OR] 7.00, 95\% CI 2.36 to 23.18; Table 1). A complex neonatal feeding type was more likely in the $22 \mathrm{q} 11.2$ deletion subgroup (OR 29.41, 95\% CI 4.53 to 134.65; Table 1). There were no significant differences for the other variables examined (Table 1).

\section{TREC values}

Term singleton samples with a 22q11.2 deletion had significantly lower median TREC values than the remaining 


\begin{tabular}{|c|c|c|c|}
\hline \multirow[b]{2}{*}{ Demographic or clinical variable } & \multicolumn{2}{|c|}{ Newborn group;* no. (\%) of newborns† } & \multirow[b]{2}{*}{ OR $(95 \% \mathrm{Cl}) \ddagger$} \\
\hline & $\begin{array}{l}\text { With 22q11.2 deletion } \\
\quad(\text { maximum } n=13)\end{array}$ & $\begin{array}{l}\text { Remaining population } \\
\text { (maximum } n=26305 \text { ) }\end{array}$ & \\
\hline Sex, male & $4(31)$ & $13467(51)$ & $0.42(0.12$ to 1.35$)$ \\
\hline \multicolumn{4}{|l|}{ Birth weight for gestational age§ } \\
\hline$<$ 10th percentile & $6(46)$ & $2869(11)$ & 7.00 (2.36 to 23.18$)$ \\
\hline$<3$ 3rd percentileף & $2(15)$ & $819(3)$ & 5.66 (0.90 to 23.80$)$ \\
\hline Neonatal transfusion ${ }^{\star *}$ & $0(0)$ & $12(0.06)$ & $0.00(0.00$ to 834.95$)$ \\
\hline Complex neonatal feeding ${ }^{\star \star} \dagger †$ & $2(18)$ & $183(0.8)$ & $29.41(4.53$ to 134.65$)$ \\
\hline Gestational age, wk, median (IQR) & 39.0 (38.0 to 39.6$)$ & 39.3 (38.5 to 40.2$)$ & $-0.3(-1.1$ to 0.2$)$ 㧊 \\
\hline Maternal age,$^{\star \star}$ yr, median (IQR) & 25.5 (24.0 to 29.5 ) & 32.0 (28.0 to 35.0$)$ & $-6.5(-7$ to -2$) \neq \ddagger$ \\
\hline \multicolumn{4}{|c|}{ 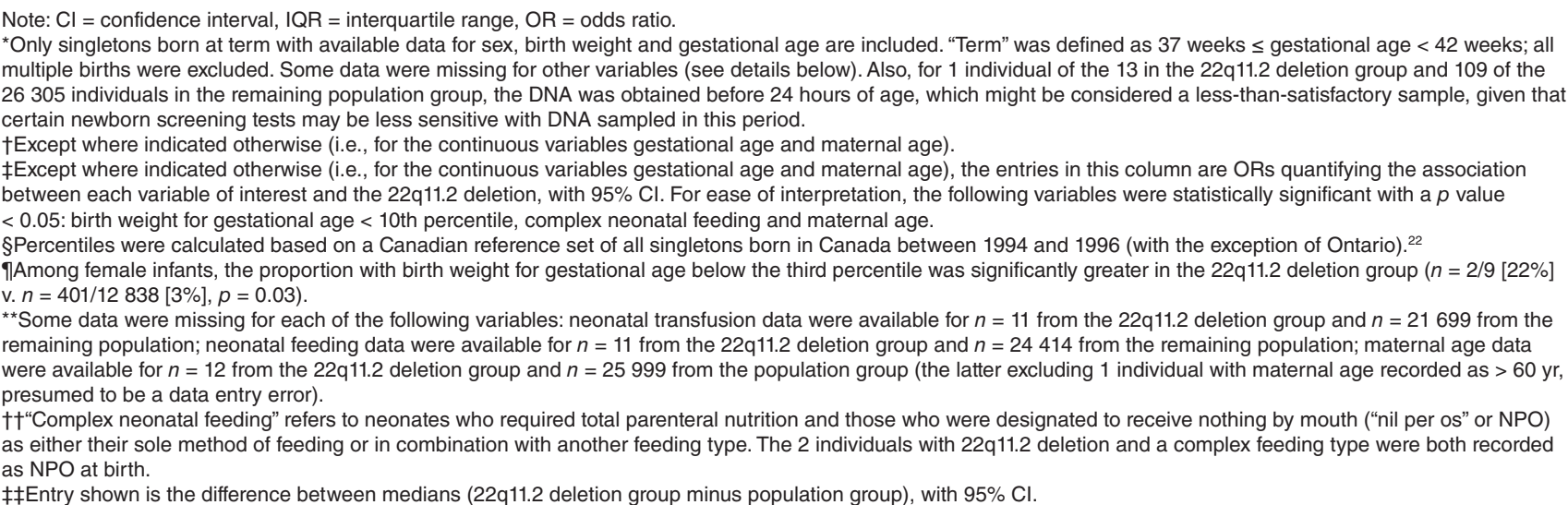 } \\
\hline
\end{tabular}

population-based samples (108.9 v. 602.5 copies/3 $\mu \mathrm{L}, p<$ $0.001)$. The proportion with fewer than 200 TREC copies/ $3 \mu \mathrm{L}$ was greater in the $22 \mathrm{q} 11.2$ deletion subgroup (Figure 2A). Six (46\%) of this 22q11.2 deletion subgroup met the initial clinical newborn screening cut-off value for severe combined immunodeficiency of 100 or fewer TREC copies/3 $\mu \mathrm{L}$ (Figure 2B), compared with 81 (0.3\%) of the population-based samples $(p<0.001)$.

For newborn screening in Ontario, all samples meeting this initial cut-off proceed to a second, confirmatory TREC assay, run in duplicate, with a cut-off of no more than 75 copies $/ 3 \mu \mathrm{L}$. Of the total 87 samples proceeding to this secondary assay, 11 met this clinical cut-off: 1 (8\%) from the $22 q 11.2$ deletion group and $10(0.04 \%)$ from the remaining population-based group $(p=0.005)$.

\section{Interpretation}

The estimated minimum prevalence of the $22 \mathrm{q} 11.2$ deletion in the Ontario newborn screening sample that we studied was 1 in 2148 (4.7 per 10000 ), with the majority of confirmed deletions spanning the full LCR22A-LCR22D region. Among singletons born at term, those with a 22 q11.2 deletion had significantly younger maternal age, lower TREC levels and a higher proportion with small birth weight for gestational age compared with the remaining population sample.

Extrapolating our results to the approximately 140000 live births annually in Ontario, ${ }^{23}$ we could expect about 66 births with a $22 q 11.2$ deletion each year. For context, comparable prevalence estimates using data from Canada and the United States for other genetic disorders (e.g., cystic fibrosis) are provided in Table 2; of these, only Down syndrome is more common than $22 \mathrm{q} 11.2 \mathrm{DS} .{ }^{18,24,25}$

The prevalence of $22 \mathrm{q} 11.2$ deletions estimated in this study is higher than previous prevalence estimates based on different sampling methods, but remains in line with the description of $22 \mathrm{q} 11.2 \mathrm{DS}$ as a rare disease (defined as $<1$ in 2000 or < 5 per 10 000). ${ }^{1} 22$ q11.2DS has historically presented a substantial diagnostic challenge for clinicians, with clinical diagnosis based on obvious congenital anomalies that do not predict the intellectual or neuropsychiatric outcomes of most concern to parents. ${ }^{1,2}$ Most affected newborns would be expected to have unaffected parents. ${ }^{1}$ However, improved pediatric care over many decades, as well as the limited effects of a $22 \mathrm{q} 11.2$ deletion on reproductive fitness when major neuropsychiatric phenotypes are absent, could lead to increasing numbers of affected parents, many of whom would be expected to be undiagnosed. ${ }^{26}$ 


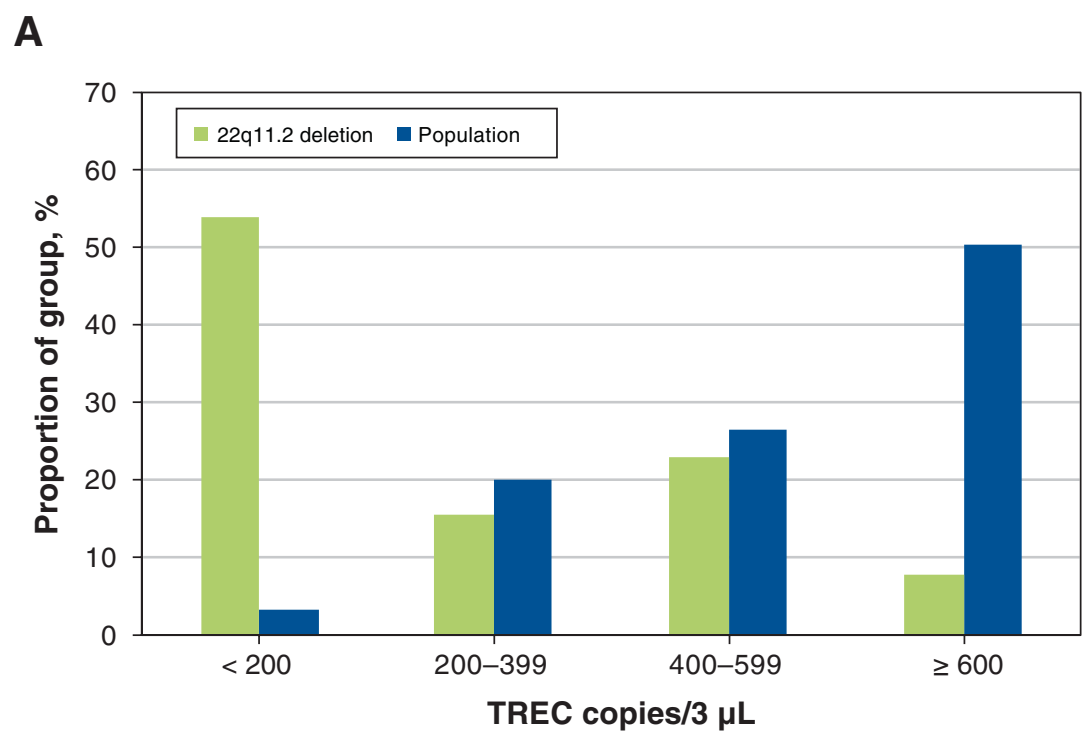

B

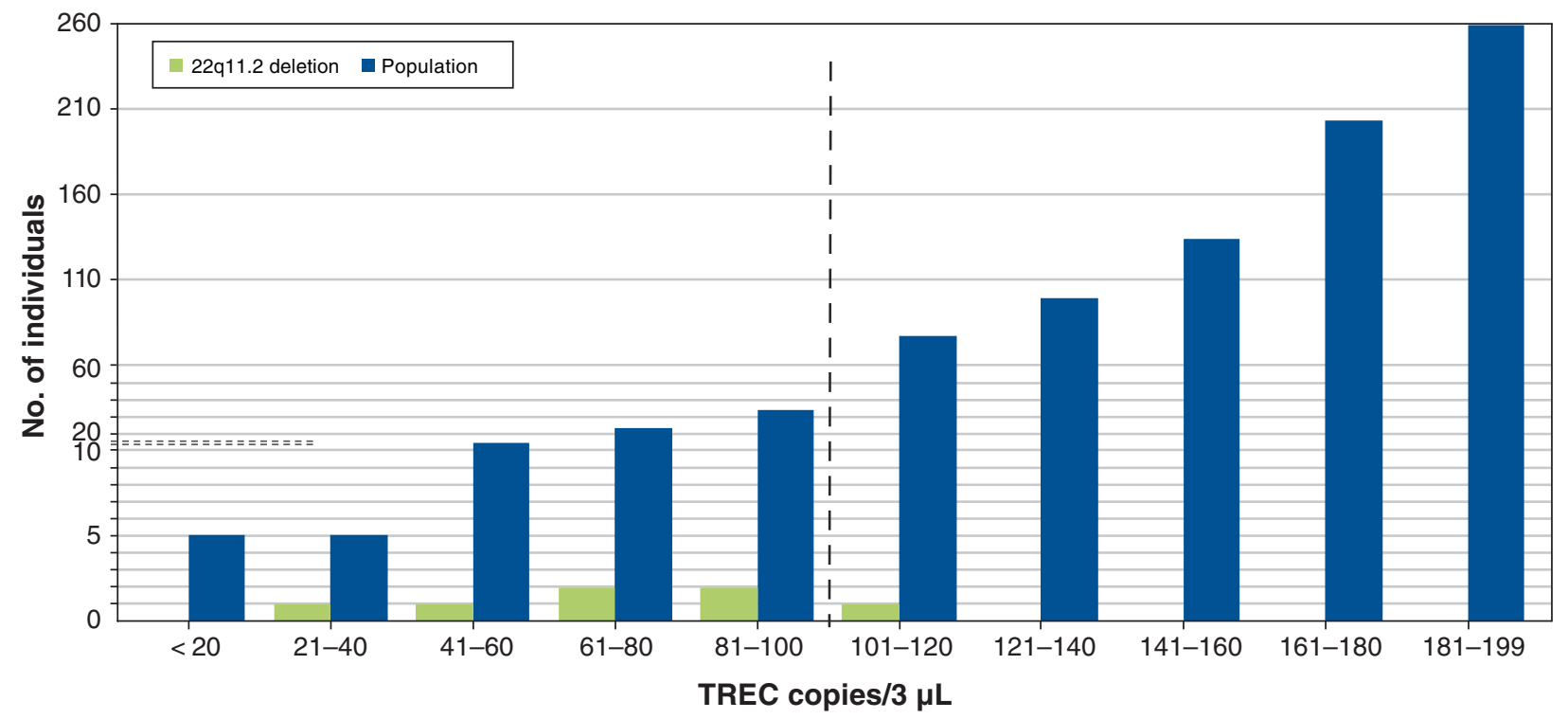

Figure 2: (A) Severe combined immunodeficiency screening results showing distribution of T-cell receptor excision circle (TREC) copies/3 $\mu \mathrm{L}$ for term singleton newborn screening samples, by 22 q 11.2 deletion status. For the term singleton subsample, the majority $(7 / 13,54 \%)$ of the 22q11.2 deletion group had fewer than 200 TREC copies/3 $\mu \mathrm{L}$ (about the 3rd percentile), whereas the greatest proportion of the remaining population-based group (13 297 [50\%] of 26 435) had at least 600 TREC copies/3 $\mu \mathrm{L}$. (B) Subset of term singleton newborn screening samples with lowest TREC values $(<200$ copies $/ 3 \mu \mathrm{L})$. Shown here are detailed distribution results for samples from the term singleton subsample. Overall, there were 859 with fewer than 200 TREC copies/3 $3 \mathrm{~L}, 7$ with confirmed $22 q 11.2$ deletion and 852 from the remaining populationbased group. The dashed horizontal lines indicate where the scale changes for fine gradations: below 10, each mark on the $y$ axis indicates 1 individual, and above 10, each mark on the $y$ axis indicates 10 individuals. Six (46\%) of the overall 13 term singleton samples with a confirmed $22 q 11.2$ deletion had no more than 100 TREC copies/3 $\mu \mathrm{L}$ (to the left of the vertical dashed line), compared with 81 (0.3\%) of the remaining population-based group $(n=26435, p<0.001)$. Currently, 100 TREC copies/3 $\mu \mathrm{L}$ is the cut-off in Ontario to undergo a secondary, more accurate TREC assay for final reporting of severe combined immunodeficiency identified by newborn screening.

In contrast to previous studies providing prevalence estimates for $22 \mathrm{q} 11.2 \mathrm{DS},{ }^{3-8,27}$ the main strength of this study was the use of an unselected contemporary newborn screening sample that was uniformly assessed using a standard method (multiplex qPCR), which is often used for newborn screening. Several previous studies used clinically ascertained samples 
Table 2: Live-birth prevalence of familiar chromosomal abnormalities and selected conditions included in newborn screening programs

\begin{tabular}{|c|c|c|}
\hline \multirow[b]{2}{*}{ Condition } & \multicolumn{2}{|c|}{$\begin{array}{l}\text { Population-based live-birth } \\
\text { prevalence }\end{array}$} \\
\hline & Estimate & Per 10000 \\
\hline \multicolumn{3}{|l|}{$\begin{array}{l}\text { Familiar chromosomal } \\
\text { abnormalities }\end{array}$} \\
\hline Trisomy $21^{*}$ & 1 in 750 & 13.33 \\
\hline Trisomy 18† & 1 in 5000 & 2.00 \\
\hline Trisomy 13† & 1 in 16000 & 0.63 \\
\hline \multicolumn{3}{|l|}{$\begin{array}{l}\text { Selected conditions included in } \\
\text { newborn screening } \ddagger\end{array}$} \\
\hline Congenital hypothyroidism & 1 in 3000 & 3.33 \\
\hline Cystic fibrosis & 1 in 3600 & 2.78 \\
\hline Phenylketonuria & 1 in 12000 & 0.83 \\
\hline SCID & $\begin{array}{l}1 \text { in } 50000 \text { to } \\
1 \text { in } 100000\end{array}$ & 0.1 to 0.2 \\
\hline \multicolumn{3}{|c|}{$\begin{array}{l}\text { Note: SCID = severe combined immunodeficiency. } \\
\text { "Based on Canadian surveillance data (excluding Quebec) from } 2005 \text { to } 2013 .{ }^{24} \\
\text { †Based on data from the US National Library of Medicine Genetics home } \\
\text { reference. } .^{25} \\
\text { †Based on approximate prevalence in Ontario. } .^{18}\end{array}$} \\
\hline
\end{tabular}

with the 22q11.2 deletion ${ }^{3-7}$ and may have underestimated the prevalence of $22 \mathrm{q} 11.2 \mathrm{DS}$, given its variable clinical phenotype, which may not include typically associated congenital anomalies. ${ }^{1}$ Only 2 previous population-based studies based their prevalence estimates solely on molecular genetic data. ${ }^{8,27}$ One was a Danish study restricted to residents 1 year of age or older, which retrospectively examined dried blood spots banked over a 24.5 -year period to $2005 ;$; that study could not account for infant mortality. ${ }^{13}$ The other was a Norwegian study restricted to newborns that required both parents to consent to participation in a genetic research study. ${ }^{27}$ The 22q11.2 deletions identified in these 2 studies represent bookends of lowest $(n=1 \text { in } 12252 \text {, or } 0.82 \text { per } 10000)^{27}$ and highest $(n=7 \text { in } 25704 \text {, or } 2.7 \text { per } 10000)^{8}$ estimated livebirth prevalence before the current study.

In the current study, as expected, the majority of $22 \mathrm{q} 11.2$ deletions spanned the full LCR22A-LCR22D region (Figure 1). ${ }^{1}$ However, rarer proximal nested $22 \mathrm{q} 11.2$ deletions comprised 3 (21\%) of the 14 confirmed 22q11.2 deletions, a higher prevalence than reported in large clinically ascertained samples. ${ }^{1,28}$ This raises the possibility that nested $22 \mathrm{q} 11.2$ deletions may have a somewhat lower penetrance for typical anatomic features leading to clinical detection ${ }^{2}$ compared with the common LCR22A-LCR22D deletion. ${ }^{28}$

In contrast to more familiar chromosomal abnormalities, such as trisomy 21 , the limited clinical data available for the samples in the current study show that pathogenic $22 \mathrm{q} 11.2$ deletions may be associated with earlier, not late, maternal age. ${ }^{29}$ Also, we found that the $22 \mathrm{q} 11.2$ deletion may be associated with mild abnormalities of fetal growth, consistent with results from a retrospective study of adults with 22q11.2DS. ${ }^{30}$

Notably, the TREC results, while consistent with previous TREC-based newborn screening studies showing a higher prevalence of the $22 \mathrm{q} 11.2$ deletion with low values,, 10 indicated that only a minority of all confirmed $22 \mathrm{q} 11.2$ deletion samples would be detected using a newborn screening strategy based on severe combined immunodeficiency. This finding was foreshadowed by the results of a previous retrospective study ${ }^{11}$ and provides further support for developing genetically based newborn screening for $22 \mathrm{q} 11.2$ deletions. For any such proposal, screening costs per newborn must be low (e.g., < US\$7 per sample ${ }^{31}$ ), methods must be scalable within current clinical newborn screening laboratories, and feasible plans for confirmatory studies and clinical referral for infants with confirmed positive screening results are needed. ${ }^{32}$

Our findings set the stage for future prospective studies to further refine prevalence estimates of high-impact 22q11.2 deletions, including the rarer proximal nested deletions. Large, multicentre newborn screening studies involving diverse jurisdictions could allow determination of factors that may affect 22q11.2DS prevalence, such as ethnicity and cultural factors, availability of prenatal screening ${ }^{33}$ and reproductive technologies. If ethics approval could be obtained, assessment of phenotypic prenatal and postnatal data, details about newborns receiving an early clinical diagnosis of 22q11.2DS, determination of inherited and de novo $22 \mathrm{q} 11.2$ deletion status and ability to provide parents of newborns with the 22q11.2DS diagnosis after clinical laboratory confirmation would offer substantially improved understanding of this important condition. A recent study of prospective mothers and previous reports would support such a study design. ${ }^{14,33}$

\section{Limitations}

The main limitation of this and other 22q11.2DS prevalence studies is the sample size. Larger, comparably ascertained samples are needed to refine live-birth prevalence estimates and improve knowledge about associated clinical features. Nonetheless, the results complement previous estimates using other designs (e.g., those based on congenital physical features and clinical recognition ${ }^{3-7}$ ) and add to studies showing high prenatal prevalence of the 22q11.2 deletion ${ }^{15}$ and strong association with fetal loss (stillbirths and miscarriages). ${ }^{34,35}$

In addition, because of restrictions related to de-identified dried blood spot samples set by the research ethics boards, minimal clinical data were available, which prevented us from learning about factors that could affect the prevalence of the $22 \mathrm{q} 11.2$ deletion or about the outcomes of these newborns, including whether and when any received a clinical diagnosis of $22 \mathrm{q} 11.2 \mathrm{DS}$.

This study used unbiased but intermittent sampling with a non-uniform distribution over a 21-month period and did not include confirmatory assays for all 30074 samples. We did not have the ability to calculate true positive and true negative rates or to evaluate the specific qPCR-based assays used, 
although we note that qPCR is already a standard method used in existing newborn screening programs. MLPA is also used in some clinical laboratories and has a sensitivity and specificity of $99 \%$ and $97 \%$, respectively, for the detection of $22 \mathrm{q} 11.2$ deletions. ${ }^{36}$

The estimate of live-birth prevalence determined in this study is referred to as a "minimum" prevalence because it could not be less but could possibly be higher. However, the results for the samples with screening results in the range of the screen-positive samples but where MLPA confirmed absence of the $22 \mathrm{q} 11.2$ deletion suggest that this would be unlikely. A well-designed and adequately powered study of newborn screening methods for $22 \mathrm{q} 11.2$ deletions is needed.

\section{Conclusion}

The results of this study provide a contemporary live-birth prevalence estimate for pathogenic $22 \mathrm{q} 11.2$ deletions that indicates $22 \mathrm{q} 11.2 \mathrm{DS}$ to be one of the most common of rare genetic conditions. The clinical findings of this study, including increased prevalence of small-for-gestational-age infants with 22q11.2 deletions and young maternal age, together with what is well known about the 22q11.2 deletion, support the public health importance of early (i.e., prenatal and neonatal) diagnosis. Early diagnosis would enable prompt screening, detection and treatment initiation for actionable features associated with 22q11.2 deletions.

\section{References}

1. McDonald-McGinn DM, Sullivan KE, Marino B, et al. 22q11.2 deletion syndrome. Nat Rev Dis Primers 2015;1:15071.

2. Palmer LD, Butcher NJ, Boot E, et al. Elucidating the diagnostic odyssey of 22q11.2 deletion syndrome. Am 7 Med Genet A 2018;176:936-44.

3. Tézenas Du Montcel S, Mendizabai H, Aymé S, et al. Prevalence of 22q11 microdeletion. 7 Med Genet 1996;33:719.

4. Botto LD, May K, Fernhoff PM, et al. A population-based study of the 22q11.2 deletion: phenotype, incidence, and contribution to major birth defects in the population. Pediatrics 2003;112:101-7.

5. Goodship J, Cross I, LiLing J, et al. A population study of chromosome 22q11 deletions in infancy. Arch Dis Child 1998;79:348-51.

6. Devriendt K, Fryns JP, Mortier G, et al. The annual incidence of DiGeorge/ velocardiofacial syndrome. 7 Med Genet 1998;35:789-90.

7. Oskarsdóttir S, Vujic M, Fasth A. Incidence and prevalence of the 22q11 deletion syndrome: a population-based study in Western Sweden. Arch Dis Child 2004;89:148-51.

8. Olsen L, Sparsø T, Weinsheimer SM, et al. Prevalence of rearrangements in the $22 \mathrm{q} 11.2$ region and population-based risk of neuropsychiatric and developmental disorders in a Danish population: a case-cohort study. Lancet Psychiatry 2018;5:573-80.

9. Martin-Nalda A, Cueto-González AM, Argudo-Ramírez A, et al. Identification of $22 \mathrm{q} 11.2$ deletion syndrome via newborn screening for severe combined immunodeficiency. Two years' experience in Catalonia (Spain). Mol Genet Genomic Med 2019;7:e1016.

10. Barry JC, Blaine Crowley T, Jyonouchi S, et al. Identification of 22q11.2 deletion syndrome via newborn screening for severe combined immunodeficiency. 7 Clin Immunol 2017;37:476-85.

11. Lingman Framme J, Borte $S$, von Döbeln U, et al. Retrospective analysis of TREC based newborn screening results and clinical phenotypes in infants with the 22q11 deletion syndrome. 7 Clin Immunol 2014;34:514-9.

12. Routes JM, Grossman WJ, Verbsky J, et al. Statewide newborn screening for severe T-cell lymphopenia. FAMA 2009;302:2465-70.

13. Repetto GM, Guzmán ML, Delgado I, et al. Case fatality rate and associated factors in patients with $22 \mathrm{q} 11$ microdeletion syndrome: a retrospective cohort study. BM7 Open 2014;4:e005041.

14. Costain G, Chow EWC, Ray PN, et al. Caregiver and adult patient perspectives on the importance of a diagnosis of $22 \mathrm{q} 11.2$ deletion syndrome. 7 Intellect Disabil Res 2012;56:641-51.

15. Grati FR, Molina Gomes D, Ferreira JCPB, et al. Prevalence of recurrent pathogenic microdeletions and microduplications in over 9500 pregnancies. Prenat Diagn 2015;35:801-9.
16. Grati FR, Gross SJ. Noninvasive screening by cell-free DNA for $22 \mathrm{q} 11.2$ deletion: benefits, limitations, and challenges. Prenat Diagn 2019;39:70-80.

17. Miller DT, Adam MP, Aradhya S, et al. Consensus statement: chromosomal microarray is a first-tier clinical diagnostic test for individuals with developmental disabilities or congenital anomalies. Am 7 Hum Genet 2010;86:749-64.

18. Newborn Screening Ontario. Newborn screening manual: a guide for newborn care providers. Edition 2.1. Ottawa: Children's Hospital of Eastern Ontario; 2018.

19. Tomita-Mitchell A, Mahnke DK, Larson JM, et al. Multiplexed quantitative real-time PCR to detect $22 \mathrm{q} 11.2$ deletion in patients with congenital heart disease. Physiol Genomics 2010;42A:52-60.

20. Spong CY. Defining 'term' pregnancy: recommendations from the Defining 'Term' Pregnancy Workgroup. FAMA 2013;309:2445-6.

21. Fay MP. Confidence intervals that match Fisher's exact or Blaker's exact tests. Biostatistics 2010;11:373-4.

22. Kramer MS, Platt RW, Wen SW, et al.; Fetal/Infant Health Study Group of the Canadian Perinatal Surveillance System. A new and improved population-based Canadian reference for birth weight for gestational age. Pediatrics 2001; 108:E35.

23. Table: 13-10-0414-01: Live births, by place of residence of mother. Ottawa: Statistics Canada. Available: https://www150.statcan.gc.ca/t1/tbl1/en/tv. action? pid=1310041401 (accessed 2020 Oct. 24).

24. Down syndrome surveillance in Canada 2005-2013. Ottawa: Public Health Agency of Canada; modified 2017 Mar. 2. Available: https://www.canada.ca/en/ public-health/services/publications/healthy-living/down-syndrome-surveillance -2005-2013.html (accessed 2020 Oct. 24).

25. Genetics [website]. Bethesda (MD): U.S. National Library of Medicine. Available: https://medlineplus.gov/genetics/ (accessed 2020 Oct. 24).

26. Costain G, Chow EWC, Silversides CK, et al. Sex differences in reproductive fitness contribute to preferential maternal transmission of $22 \mathrm{q} 11.2$ deletions. 7 Med Genet 2011:48:819-24.

27. Smajlagić D, Lavrichenko K, Berland S, et al. Population prevalence and inheritance pattern of recurrent CNVs associated with neurodevelopmental disorders in 12,252 newborns and their parents. Eur 7 Hum Genet 2021;29:205-15.

28. Guo T, Diacou A, Nomaru H, et al.; International Chromosome 22q11.2; International 22q11.2 Brain and Behavior Consortia. Deletion size analysis of 168022 q11.2DS subjects identifies a new recombination hotspot on chromosome 22q11.2. Hum Mol Genet 2018;27:1150-63.

29. Delio M, Guo T, McDonald-McGinn DM, et al. Enhanced maternal origin of the $22 \mathrm{q} 11.2$ deletion in velocardiofacial and DiGeorge syndromes. Am 7 Hum Genet 2013;92:439-47.

30. Van L, Butcher NJ, Costain G, et al. Fetal growth and gestational factors as predictors of schizophrenia in 22q11.2 deletion syndrome. Genet Med 2016;18:350-5.

31. Wells J, Rosenberg M, Hoffman G, et al. A decision-tree approach to cost comparison of newborn screening strategies for cystic fibrosis. Pediatrics 2012;129:e339-47.

32. Wilson JM, Jungner G. Principles and practice of screening for disease [article in Spanish]. Bol Oficina Sanit Panam 1968;65:281-393.

33. Kagan KO, Hoopman M, Pfaff T, et al. First trimester screening for common trisomies and microdeletion $22 \mathrm{q} 11.2$ syndrome using cell-free DNA: a prospective clinical study. Fetal Diagn Ther 2020;47:841-52.

34. Maisenbacher MK, Merrion K, Pettersen B, et al. Incidence of the $22 \mathrm{q} 11.2$ deletion in a large cohort of miscarriage samples. Mol Cytogenet 2017;10:6.

35. Reddy UM, Page GP, Saade GR, et al.; NICHD Stillbirth Collaborative Research Network. Karyotype versus microarray testing for genetic abnormalities after stillbirth. N Engl 7 Med 2012;367:2185-93.

36. Vorstman JAS, Jalali GR, Rappaport EF, et al. MLPA: a rapid, reliable, and sensitive method for detection and analysis of abnormalities of $22 \mathrm{q}$. Hum Mutat 2006;27:814-21.

Affiliations: Clinical Genetics Research Program (Blagojevic, Heung, Bassett), Centre for Addiction and Mental Health; The Dalglish Family 22q Clinic (Blagojevic, Heung, Bassett), University Health Network; Department of Psychiatry (Bassett), University of Toronto, Toronto, Ont.; Children's Hospital of Eastern Ontario Research Institute (Theriault, Chakraborty, Kernohan, Bulman), University of Ottawa, Ottawa, Ont.; Division of Pediatric Cardiothoracic Surgery (TomitaMitchell), Department of Surgery, Medical College of Wisconsin, Herma Heart Institute, Milwaukee, Wisc.

Contributors: Anne Bassett, Dennis Bulman and Aoy Tomita-Mitchell were involved in conceptualization and design of the study. Mylene Theriault, Kristin Kernohan and Pranesh Chakraborty were involved in data acquisition. Mylene Theriault, Christina Blagojevic and Tracy Heung were involved in data analysis. Christina Blagojevic, Tracy Heung and Anne Bassett were involved in interpretation of the data. Christina Blagojevic drafted the manuscript, which was critically revised primarily by Anne Bassett with contributions from all other authors. All of the authors provided approval of the manuscript for publication and agreed to be accountable for the work. 
Funding: This work was funded by a McLaughlin Centre Accelerator Grant (Anne Bassett, Dennis Bulman), with support from Canadian Institutes of Health Research grant PJT-148924 (Anne Bassett) and, for prior work relevant to probe development, a National Institute of Child Health and Human Development grant 1R21-HD-060309-01 and funding from the Audrey Hohenwalter VCFS Foundation (Aoy Tomita-Mitchell). Anne Bassett holds the Dalglish Chair in 22q11.2 Deletion Syndrome at the University Health Network and the University of Toronto.

Content licence: This is an Open Access article distributed in accordance with the terms of the Creative Commons Attribution (CC BY-NC-ND 4.0) licence, which permits use, distribution and reproduction in any medium, provided that the original publication is properly cited, the use is noncommercial (i.e., research or educational use), and no modifications or adaptations are made. See: https://creativecommons.org/licenses/ by-nc-nd/4.0/

Data sharing: Data sharing is precluded as per stipulations of the research ethics boards that approved the study.
Acknowledgements: The authors thank personnel of the Newborn Screening Ontario program at the Children's Hospital of Eastern Ontario for sample collection and laboratory investigations, as well as personnel affiliated with the Clinical Genetics Research Program at the Centre for Addiction and Mental Health (CAMH) and the Dalglish Family 22q Clinic at the Toronto General Hospital for their assistance with data checking and clinical context. The authors thank Marcos Sanches, biostatistician at CAMH, for assistance with the statistical analyses. They also thank individuals involved in the original conceptualization and probe testing, as well as colleagues, patients and family members whose initiative and motivation have inspired efforts to develop newborn screening for $22 \mathrm{q} 11.2$ deletion syndrome. The authors also acknowledge those who attended and supported the initiative to introduce newborn screening for 22q11.2 deletion syndrome to the Secretary's Advisory Committee on Heritable Disorders in Newborns and Children in Washington, DC, in 2012.

Supplemental information: For reviewer comments and the original submission of this manuscript, please see www.cmajopen.ca/content/9/3/ E802/suppl/DC1. 